\title{
New perspectives on Bordetella pathogenicity
}

\section{New species}

Until recently, only three species of Bordetella were recognised: $B$. pertussis, the cause of whooping cough; $B$. parapertussis associated with mild whooping cough; and $B$. bronchiseptica, an important respiratory pathogen in wild and domesticated animals that occasionally affects man. In 1984, B. avium was proposed as an agent of respiratory disease of turkey poults and other birds [l]. Two new species have now been added to the genus on the basis of DNA hybridisation studies, DNA base ratio determinations and other genotypic and phenotypic characteristics. The species $B$. holmesii was proposed [2] for a group of 15 strains isolated from blood cultures of patients, some of whom were immunocompromised. $B$. hinzii was the name given to a $B$. avium-like group of organisms from the respiratory tracts of turkeys and chickens [3]; attempts to produce disease experimentally were unsuccessful. Two strains have been obtained from man, one from sputum and one from the blood of an AIDS patient. It is likely that other 'atypical' bordetellae will be reported in the near future.

\section{New habitats}

The bordetellae are usually defined as obligate, noninvasive parasites of the respiratory tracts of warmblooded animals, including birds, with a predilection for the respiratory ciliated epithelium. This definition has been confounded by a number of recent observations. Firstly, several species, including the newly described $B$. holmesii, have been isolated from human blood, mainly from immunocompromised patients. Secondly, it has become apparent that $B$. pertussis, $B$. parapertussis and $B$. bronchiseptica have mechanisms for attachment to, invasion of and survival in, both epithelial cells and phagocytes. The significance of this is not yet clear but it may be important in the protracted course of bordetella infections or in the establishment of a quiescent or chronic stage. The importance of cell-mediated immunity of $B$. pertussis infection, demonstrated in animal models, may be due in part to the destruction of such infected cells [4]. Thirdly, B. bronchiseptica and B. avium have been shown to survive and grow in lakewater and phosphatebuffered saline and these species could perhaps exist as sources of infection in a freshwater habitat [5]. Fourthly, the ability of Bordetella spp. to regulate virulence factor expression in response to external signals [6] strongly suggests that they have alternative habitats, either in vivo or ex vivo, where such regulation would be an advantage. Until recently, $B$. parapertussis was considered to be a purely human parasite, like $B$. pertussis. Its isolation from healthy and pneumonic lambs has changed this view and may have identified a reservoir of infection for man [7].

\section{New perspectives on virulence factors}

The bordetellae produce an array of toxins, aggressins and adhesins that are presumed to be important in colonisation of their respective hosts and ensuring their survival and propagation (Table 1). Such information

Table 1. Virulence factors of Bordetella species*

\begin{tabular}{|c|c|c|c|c|c|}
\hline Virulence factor & B. pertussis & B. parapertussis & B. bronchiseptica & B. avium & Probable role in pathogenicity \\
\hline Tracheal cytotoxin & + & + & + & + & Ciliostasis, cytotoxicity \\
\hline Heat-labile toxin & + & + & + & + & Local inflammatory effects, cytotoxicity \\
\hline Fimbriae & + & + & + & + & Adhesion, invasion \\
\hline Filamentous haemagglutinin & + & + & + & - & Adhesion, invasion \\
\hline Adenylate cyclase toxin & + & + & + & - & Interference with immune effector cells \\
\hline Pertactin & + & + & + & NK & Adhesion, invasion \\
\hline BrkA & + & $+t$ & $+\dagger$ & - & Adhesion, invasion, serum resistance \\
\hline Tracheal colonisation factor & + & - & - & NK & Adhesion \\
\hline Pertussis toxin & + & - & - & - & $\begin{array}{l}\text { Adhesion, invasion, interference } \\
\text { with immune effector cells }\end{array}$ \\
\hline Osteotoxin & - & - & - & + & Cytotoxicity \\
\hline
\end{tabular}

NK, not known.

*Data from Parton [15].

†Sequences homologous to the $B$. pertussis brk gene are present in B. parapertussis and B. bronchiseptica.

†Gentry-Weeks et al. [16]. 
may provide clues as to the factors responsible for the unique or common features of the bordetella diseases, which include: age-related susceptibility to infection; adherence of organisms to ciliated epithelium and loss of ciliated cells from the respiratory tract; weight gain of the host; excessive mucus production; and some form of cough. A predisposition to secondary infections is also common and this increases the mortality in untreated cases.

Tracheal cytotoxin (TCT) and heat-labile dermonecrotising toxin (HLT) are likely to be important in the pathogenesis of bordetella infections as they are produced by all four species. TCT was originally identified by its ability to cause ciliostasis and ciliated cell extrusion in hamster tracheal organ cultures. The selective destruction of ciliated cells is similar to that seen in necropsy material from human pertussis and in turkey coryza. Such effects could account for some of the pathological events of bordetella respiratory infections such as accumulation of mucus, coughing and predisposition to secondary infections. This unusual toxin belongs to a family of muramyl peptides with diverse biological activities such as pyrogenicity, adjuvanticity, arthritogenicity, induction of slow-wave sleep and stimulation of IL-1 production. Recent work has implicated nitric oxide, synthesised in response to IL-1, as the actual cytotoxic factor [8]. TCT appears to have a central role in atrophic rhinitis, an economically important disease of swine, characterised by inflammation and atrophy of the nasal turbinates, snout deformation and retarded growth. Although $B$. bronchiseptica alone can cause mild to moderate turbinate atrophy, the severe, progressive disease appears to be caused by subsequent colonisation by toxigenic Pasteurella multocida. TCT released by $B$. bronchiseptica may enhance colonisation by $P$. multocida by inducing ciliostasis and mucus accumulation [9]. Both species produce dermonecrotising toxins that, on repeated local or parenteral administration, cause progressive turbinate atrophy.

Apart from a probable role in producing turbinate atrophy associated with atrophic rhinitis, the role of HLT in bordetella diseases is ill-defined. HLT preparations have potent vasoconstrictive activity and in experimental animals cause death or loss of normal weight gain, spleen atrophy and ischaemic lesions or necrosis of skin. Despite its potency in various bioassays, $B$. pertussis mutants deficient in HLT production are unaltered in their ability to cause a lethal infection in mice. Recent sequence analysis has revealed homology between $B$. pertussis HLT and $E$. coli CNF1 (cytotoxic necrotising factor 1) toxin [10].

Pertussis toxin (PT) has a remarkably diverse range of biological activities and an assumed central role in the pathogenesis of whooping cough. However, as PT is unique to $B$. pertussis, it is difficult to assign it a preeminent role. This view has been strengthened by two recent studies in which paroxysmal coughing, whooping and vomiting were all features of parapertussis infections, with frequencies approaching those in pertussis cases $[11,12]$. The main difference was that lymphocytosis, due to $\mathrm{PT}$, was not a feature of parapertussis. These findings suggest that PT plays a minor role, if any, in causing the typical symptoms of whooping cough.

Two new virulence factors of $B$. pertussis, namely BrkA [13] and tracheal colonisation factor (Tcf) [14], are of particular interest because of their close structural homology with pertactin (Table 1) and with other bacterial proteins involved in eukaryotic cell interactions, e.g., SepA, thought to be involved in tissue invasion by Shigella flexneri [17]. B. pertussis pertactin has been shown to mediate attachment of the bacterium to, and invasion of, various cell lines and attachment involves an Arg-Gly-Asp (RGD) integrinbinding domain. BrkA and Tcf similarly act as adhesins and contain RGD sequences. All three are synthesised as large precursor molecules and processed proteolytically into the $\mathrm{N}$-terminal mature proteins and C-terminal fragments which appear to be necessary for expression of the mature proteins at the cell surface.

\section{New pertussis vaccines}

Toxoided PT (PTd) has become the prime candidate antigen for acellular pertussis vaccines. In field trials, a mono-component PTd vaccine and a two-component vaccine containing PTd and filamentous haemagglutinin (FHA) were less protective against culture or serologically confirmed pertussis than would have been expected for a whole-cell vaccine [18]. However, both vaccines gave good protection against severe disease and provided evidence that PTd alone or with other antigens could make effective vaccines. Formaldehyde detoxification of PT reduces its immunogenicity and more promising vaccines, containing PT toxoided with hydrogen peroxide or recombinant PTd, are now available. Further trials are in progress to compare other vaccine formulations containing combinations of PTd or recombinant PTd, FHA, pertactin and agglutinogens (fimbrial antigens). Preliminary results suggest that the multi-component vaccines, especially those containing pertactin, are highly efficacious and show few adverse effects [19]. Pertactin is known to protect mice against aerosol challenge with $B$. pertussis and, with FHA, against intracerebral challenge. It was also present in significant amounts as a contaminant in one of the early Japanese acellular vaccines.

\section{R. PARTON}

Division of Infection and Immunity,

Joseph Black Building,

University of Glasgow, Glasgow G12 8QQ 


\section{References}

1. Kersters K, Hinz K-H, Hertle A et al. Bordetella avium sp. nov., isolated from the respiratory tracts of turkeys and other birds. Int J Syst Bacteriol 1984; 34: 56-70.

2. Weyant RS, Hollis DG, Weaver RE et al. Bordetella holmesii sp. nov., a new gram-negative species associated with septicemia. J Clin Microbiol 1995; 33: 1-7.

3. Vandamme $\mathrm{P}$, Hommez J, Vancanneyt $\mathrm{M}$ et al. Bordetella hinzii sp. nov., isolated from poultry and humans. Int J Syst Bacteriol 1995; 45: 37-45.

4. Redhead K, Watkins J, Barnard A, Mills KHG. Effective immunization against Bordetella pertussis respiratory infection in mice is dependent on induction of cell-mediated immunity. Infect Immun 1993; 61: 3190-3198.

5. Porter JF, Wardlaw AC. Long-term survival of Bordetella bronchiseptica in lakewater and in buffered saline without added nutrients. FEMS Microbiol Lett 1993; 110: 33-36.

6. Stibitz S, Miller JF. Coordinate regulation of virulence in Bordetella pertussis mediated by the vir (bvg) locus. In: Miller VL, Kaper JB, Portnoy DA, Isberg RR (eds) Molecular genetics of bacterial pathogenesis. Washington DC, American Society for Microbiology. 1994: 407-422.

7. Porter JF, Connor K, Donachie W. Isolation and characterization of Bordetella parapertussis-like bacteria from ovine lungs. Microbiology 1994; 140: 255-261.

8. Heiss LN, Lancaster JR, Corbett JA, Goldman WE. Epithelial autotoxicity of nitric oxide: role in the respiratory cytopathology of pertussis. Proc Natl Acad Sci USA 1994; 91: 267-270.

9. Dugal F, Belanger M, Jacques $M$. Enhanced adherence of Pasteurella multocida to porcine tracheal rings preinfected with Bordetella bronchiseptica. Can J Vet Res 1992; 56: 260-264.
10. Walker KE, Weiss AA. Characterization of the dermonecrotic toxin in members of the Genus Bordetella. Infect Immun 1994; 62: 3817-3828.

11. Heininger U, Stehr $\mathrm{K}$, Schmitt Grohe $\mathrm{S}$ et al. Clinical characteristics of illness caused by Bordetella parapertussis compared with illness caused by Bordetella pertussis. Pediatr Infect Dis $J$ 1994; 13: 306-309.

12. Wirsing von Konig $\mathrm{CH}$, Finger $\mathrm{H}$. Role of pertussin toxin in causing symptoms of Bordetella parapertussis infection. Eur $J$ Clin Microbiol Infect Dis 1994; 13: 455-458.

13. Fernandez RC, Weiss AA. Cloning and sequencing of a Bordetella pertussis serum resistance locus. Infect Immun 1994; 62: 4727-4738.

14. Finn TM, Stevens LA. Tracheal colonization factor: a Bordetella pertussis secreted virulence determinant. Mol Microbiol 1995; 16: 625-634.

15. Parton R. Bordetella. In: Collier L (ed) Topley and Wilson's Principles of microbiology and microbial infections, vol 2. London, Edward Arnold. 1996 (in press).

16. Gentry-Weeks CR, Keith JM, Thompson J. Toxicity of Bordetella avium beta-cystathionase toward MC3t3-El osteogenic cells. J Biol Chem 1993; 268: 7298-7314.

17. Benjelloun-Touimi Z, Sansonetti PJ, Parsot C. SepA, the major extracellular protein of Shigella flexneri: autonomous secretion and involvement in tissue invasion. Mol Microbiol 1995; 17: 123-135.

18. Ad hoc group for the study of pertussis vaccines. Placebocontrolled trial of two acellular pertussis vaccines in Sweden. Protective efficacy and adverse events. Lancet 1988; 1: 955960.

19. Marwick C. Acellular pertussis vaccine hailed for infants. JAMA 1995; 274: 446-447. 